\title{
Recent Progress in GW-Invariants of Calabi-Yau Threefolds
}

\author{
Jun Li
}

The last two decades is a period when exchange of ideas between algebraic geometry and theoretical physics has created several fast advancing branches in algebraic geometry. The research on Gromov-Witten (GW-) invariants is a notable example of such branches.

GW-invariants is a mathematical foundation to the type II Super-String theory. In terms of algebraic geometry, it is a "virtual" counting of holomorphic maps from algebraic curves to a projective variety. Via a multiple cover relation, the GW-invariants will provide a "virtual" counting of holomorphic embedded curves.

Properties of GW-invariants differ according to the kinds of the varieties. One indicator is the Kodaira dimensions. The Theory of GWinvariants of Fano varieties is the complete opposite of those with positive Kodaira dimensions, the former is much richer than the later. However, the GW-invariants of Calabi-Yau varieties are the most mysterious and challenging. In this note, we will comment on the recent progress in research on the GW-invariants of Calabi-Yau threefolds. Due to the interest of the author, we will only report on progress via algebraic geometry approach.

The investigation of GW-invariants went through several periods of development. Inspired by the $\sigma$-model in Super-String theory [37] and using pseudo-holomorphic maps introduced by Gromov, Ruan-Tian [32] constructed the GW-invariants of semi-positive, including Calabi-Yau, symplectic manifolds. Later, Li-Tian [23] and Behrend-Fantechi [5, 4] constructed the GW-invariants of all smooth projective varieties via algebraic geometry. Their construction is based on the construction of virtual cycles of the moduli of Kontsevich's stable morphisms, and the GWinvariants are the degree of these cycles paired with tautological classes of the moduli space. The symplectic construction of GW-invariants was completed by the work of Fukaya-Ono [9] and Li-Tian [24], Ruan [31] and Siebet [33]; also a much detailed technical account by Zinger [42]. The equivalence 
of the algebraic and the symplectic construction was proved by Li-Tian [25] and by Siebert [34]. This concludes the foundation of the GW-invariants.

By the time the rigorous construction of the GW-inariants is completed, several groups had already made progress in uncovering the structure of the GW-invariants of Calabi-Yau threefolds. The first major advancement was from the mirror symmetry by Super-String theorists on the genus zero GW-invariants of Calabi-Yau threefolds: many of such invariants can be effectively be computed by studying the variation of Hodge structures of the mirror Calabi-Yau threefolds at large complex structure limit. The first striking example is that of the quintic Calabi-Yau threefolds in $\mathbb{P}^{4}$ by Candelas-Ossa-Green-Parkes [7]. Shortly after, more and more were computed. This was an exciting period. Unlike the term-by-term approach that was typical for problems of that complexity, one can explicitly and effectively find closed formulas of the generating functions of all degree genus zero GW-invariants for a large class of Calabi-Yau threefolds.

That brought the task of mathematically proving these formulas to the forefront. Though the mirror symmetry is effective in providing convincing closed forms for the genus zero GW-invariants, mathematical proofs are necessary to confirm and to further this circle of ideas. Two effective methods sprung out this urge: one is the hyperplane property of the $\mathrm{GW}$-invariants and the other is the localization by group actions.

In his paper, Kontsevich [16] proposed to use a hyperplane theorem of genus zero GW-invariants to relate that of a smooth Calabi-Yau quintic with that of $\mathbb{P}^{4}$, and then evaluate them using localization via the $\mathbb{C}^{*}$-action on $\mathbb{P}^{4}$. This combination transforms the genus zero GW-invariants into a sum formula over a set of combinatoric data. Much later, the genus zero formula of Candelas for quintics was proved by the independent work of Givental [10] and Lian-Liu-Yau [26].

For higher genus GW-invariants, based on the Kodaira-Spencer theory of gravity developed by Bershadsky-Cecotti-Ooguri-Vafa [6], genus one and more GW-invariants of quintics were derived. On the other hand, mathematically little progress is made on high genus GW-invariants of quintics, let along other closed Calabi-Yau threefolds. The main obstacle is the lack of an analogue hyperplane property for high genus GW-invariants of Calabi-Yau complete intersection in projective spaces.

Recently, Li-Zinger [22] proved the hyperplane formula for genus one reduced GW-invariants of complete intersection Calabi-Yau threefolds and proved a splitting formula relating the genus one GW-invariants in terms of the genus zero GW-invariants and the reduced genus one $\mathrm{GW}$-invariants. This is the generalization of the hyperplane property of the genus zero GW-invariants of complete intersections.

Based on this hyperplane property of the reduced genus one invariants, Zinger [40] proved the conjectural generating function of genus one GW-invariants of quintic Calabi-Yau threefolds. 
Another direction of progress on GW-invariants is for toric Calabi-Yau threefold. In this direction, following the lead of the topological vertex theory of Aganagic-Klemm-Marino-Vafa [1], Li-Liu-Liu-Zhou developed a mathematical treatment of such theory [20] using moduli of relative stable morphisms to formal toric Calabi-Yau threefolds. The last remaining step of their treatment was recently completed by the work of Maulik-OblomkovOkounkv-Pandharipande [29].

Another topic we will touch on is the recent progress toward GopakumarVafa conjectrue using Donaldson-Thomas invariants. This opens a way to use moduli of sheaves to study GW-invariants of Calabi-Yau threefolds.

\section{GW-invariants of Calabi-Yau threefolds}

Let $X$ be a smooth projective variety. For a homology class $d \in H_{2}(X, \mathbb{Z})$ and integers $g, n$, one forms the moduli of stable morphisms from genus $g$, $n$-pointed nodal curves to $X$ of fundamental class $d$ :

$$
\overline{\mathcal{M}}_{g, n}(X, d)=\left\{f: C \rightarrow X, p_{1}, \cdots, p_{n} \in C: f_{*}([C])=d, f \text { stable }\right\} / \sim .
$$

Here a morphism $f$ is stable if there are at most finitely many automorphisms of the domain that fixes the marked points and leaves $f$ invariants.

The moduli space $\overline{\mathcal{M}}_{g, n}(X, d)$ admits an obvious obstruction theory, and is perfect. Thus according to the construction of virtual cycles developed for this purpose by Li-Tian [23] and Behrend-Fantechi $[\mathbf{5}, \mathbf{4}]$, it has a virtual fundamental class

$$
\left[\overline{\mathcal{M}}_{g, n}(X, d)\right]^{\mathrm{vir}} \in H_{2 \nu}\left(\overline{\mathcal{M}}_{g, n}(X, d), \mathbb{Q}\right),
$$

of degree twice of the virtual dimension of $\overline{\mathcal{M}}_{g, n}(X, d)$ :

$$
\nu=(g-1)(3-\operatorname{dim} X)+d \cdot c_{1}(X)+n .
$$

The full GW-invariants of $X$ is defined by pairing the virtual cycle with the tautological classes of $\overline{\mathcal{M}}_{g, n}(X, d)$. First, the moduli space $\overline{\mathcal{M}}_{g, n}(X, d)$ admits two tautological morphisms: one evaluation and the other forgetful:

$$
e v: \overline{\mathcal{M}}_{g, n}(X, d) \longrightarrow X^{n} \quad \text { and } \quad \pi: \overline{\mathcal{M}}_{g, n}(X, d) \longrightarrow \overline{\mathcal{M}}_{g, n} .
$$

Pulling back cohomology classes of $X$ via the evaluation map ev provides the first set of tautological classes on $\overline{\mathcal{M}}_{g, n}(X, d)$; pulling back the classes on $\overline{\mathcal{M}}_{g, n}$ via $\pi$ provides the second set of tautological classes. The numerical GW-invariants (with insertions) are

$$
\int_{\left[\overline{\mathcal{M}}_{g, n}(X, d)\right]^{\mathrm{vir}}} e v^{*}\left(\tau_{1} \cdots \tau_{n}\right) \cdot \pi^{*}\left(c_{1}\left(\psi_{1}\right)^{k_{1}} \cdots \pi^{*} c_{1}\left(\psi_{n}\right)^{k_{n}}\right) .
$$

For $X$ a Calabi-Yau threefold, one notes that the virtual dimension of $\overline{\mathcal{M}}_{g}(X, d)$ (in case $n=0$ ) are zero regardless of the choices of $g$ and $d$. Thus the cycles $\left[\overline{\mathcal{M}}_{g, n}(X, d)\right]^{\text {vir }}$ are dimension zero classes; taking their degrees, 
we obtain the degree $d$ genus $g \mathrm{GW}$-invariants of $X$ :

$$
N_{g}^{d}=\operatorname{deg}\left[\overline{\mathcal{M}}_{g}(X, d)\right]^{\text {vir }} \in \mathbb{Q} .
$$

Since $\overline{\mathcal{M}}_{g}(X, d)$ is a DM-stack, the degree is a rational number.

The GW-invariants $N_{g}^{d}$ form a generating function (as a formal power series)

$$
F_{X}(\lambda, q)=\sum F_{X, g}(q) \lambda^{2 g-2}=\sum N_{g}^{d} \lambda^{2 g-2} q^{d}
$$

The structure of this series is the focus of this note.

\section{The conjectures from Super-String theories}

To motivate the subject, let us first recall a few outstanding conjectures on GW-invariants of Calabi-Yau threefolds from mathematical physics. To keep focused, we will only bring up the mirror symmetry and its generalization of Calabi-Yau threefolds, the Goparkumar-Vafa conjecture on the BPS states of Calabi-Yau threefolds, and the topological vertex theory of the toric Calabi-Yau threefolds.

Mirror symmetry is about the equivalence of two Super-String theories associated to mirror pairs of Calabi-Yau varieties. Its early application to GW-invariants provided the prediction of the genus zero invariants for quintic CY-threefolds in $\mathbf{P}^{4}$ by Candelas et al. [7]. Later, it was generalized to the high genus case by Vafa et al. [6]. In short, given a pair of mirror CY-threefolds $X$ and $X^{\prime}$, one can form a smooth section $\mathcal{F}_{X}$ of the tautological line bundle $\mathcal{L}^{2-2 g}$ on the moduli of deformations of the complex structure of $X$; for the mirror $X^{\prime}$ one takes the $F_{X^{\prime}}$ defined using the GW-invariants of $X^{\prime}$, viewed as a function over the space of complexified Kahler classes. The BCOV theory (a generalization of mirror symmetry) states that the section $\mathcal{F}_{X}$ satisfies an equation that makes it uniquely determined up to a finite dimensional freedom; the limiting behavior of $\mathcal{F}_{X}$ recovers $F_{X^{\prime}}$. Based on this reasoning, low genus GW-invariants of quintic CY-threefolds can be explicitly determined.

Equally important is the notion of "virtual embedded curves" in a CalabiYau threefold. Unlike the GW-invariants that take values in $\mathbb{Q}$, the "virtual" number of curves are integer valued. Drawing insights from Super-String theories, Goparkumar-Vafa $[\mathbf{1 1}]$ conjectured that the "virtual" number $n_{g}^{d}$ of curves of genus $g$ and class $d$ in a Calabi-Yau threefold $X$ are related to the GW-invariants $N_{g}^{d}$ via

$$
F_{X}=\sum_{d, g} N_{g}^{d} \lambda^{2 g-2} q^{d}=\sum_{d, g, k} \frac{n_{g}^{d}}{k}\left(2 \sin \frac{k \lambda}{2}\right)^{2 g-2} q^{k d} .
$$

Notice that collecting the coefficients of $\lambda^{-2}$, one derives the multiple cover formula of Morrison-Plesser in the case of $g=0[\mathbf{2 7}]$.

For toric Calabi-Yau threefolds, the theory of topological vertex is an effective theory on their all genus GW-invariants. In [1], Aganagic-KlemmMarino-Vafa developed a theory computing the GW-invariants of toric 
Calabi-Yau threefolds in all genera and all degrees. Their theory is based on the open Gromov-Witten invariants and on a physically derived duality between Chern-Simons theory and Gromov-Witten theory that the topological vertex can be expressed in terms of the explicitly computable Chern-Simons link invariants. Then by a gluing algorithm, they derived an algorithm computing all genera Gromov-Witten invariants of toric CalabiYau threefolds.

In recent years, we have seen progress on GW-invariants on the above mentioned directions. The mathematical theory of the topological vertex theory on GW-invariants of toric Calabi-Yau threefolds was developed; the hyperplane property of the genus one GW-invariants of complete intersection Calabi-Yau threefolds was established; the conjectural mathematical definition of the "virtual" number of embedded curves in Calabi-Yau threefolds was formulated. We are at the beginning of a fast pace progress on the GW-invariants of Calabi-Yau threefolds.

In the remainder of this note, we will go through these developments.

\section{All genera GW-invariants of toric Calabi-Yau threefolds}

The all genera GW-invariants of toric Calabi-Yau threefolds have been completely solved by the combined work of Aganagic-Klemm-Marino-Vafa [1], Li-Liu-Liu-Zhou [20] and Maulik-Oblomkov-Okounkv-Pandharipande $[29]$.

3.1. We begin with a brief outline of the topological vertex theory of AKMV. The starting point is the existence of certain open GW invariants that count holomorphic maps from bordered Riemann surfaces to $\mathbb{C}^{3}$ with boundaries mapped to three specific Lagrangian submanifolds $L_{1}, L_{2}$ and $L_{3}$. Such invariants depend on the date that include (1) the topological type of the domains classified by the genus and the number of boundary circles; (2) the topological type of the maps characterized by a triple of partitions $\vec{\mu}=\left(\mu^{1}, \mu^{2}, \mu^{3}\right)$ that keeps track of the "winding numbers" of the boundary circles in $L_{i} \cong S^{1} \times \mathbb{C}$; (3) by the "framing" $n_{i} \in \mathbb{Z}$ of the Lagrangian submanifolds $L_{i}$.

The topological vertex

$$
C_{\vec{\mu}}(\lambda ; \mathbf{n})
$$

is a generating function in indeterminant $\lambda$ and indexed by $\vec{\mu}$ and $\mathbf{n}$ after summing the invariants over the genus of the domains.

By a duality between Chern-Simons theory and GW-invariant theory in Super-String theory, Vafa et al. demonstrated that the topological vertex is given in terms of $\mathcal{W}_{\vec{\mu}}(q)$, a combinatorial expression of the Chern-Simons link invariants, in the simple form

$$
C_{\vec{\mu}}(\lambda ; \mathbf{n})=q^{\frac{1}{2}\left(\sum_{i=1}^{3} \kappa_{\nu^{i}} n_{i}\right)} \mathcal{W}_{\vec{\mu}}(q), \quad q=e^{\sqrt{-1} \lambda} .
$$

For general toric Calabi-Yau threefold, its GW invariants can be expressed in terms of $C_{\vec{\mu}}(\lambda ; \mathbf{n})$ by explicit gluing algorithms. Then by deriving explicitly 
the Chern-Simons link invariants, this theory effectively computes all GW-invariants of toric Calabi-Yau threefolds.

This is a beautiful and powerful theory; it is also a mystery to mathematicians why such a theory should exist. To carry out the program mathematically, along the strategy given by the work of topological vertex, one needs to develop a theory of open string GW-invariant theory, find degeneration of a general toric Calabi-Yau to a union of elementary ones, prove a degeneration formula and then relate that of the elementary one to ChernSimons link invariants.

3.2. The approached developed in $[\mathbf{2 0}]$ is to define the topological vertex $C_{\vec{\mu}}(\lambda ; \mathbf{n})$ via algebraic geometry using moduli of relative stable morphisms developed $[\mathbf{1 8}, \mathbf{1 9}, \mathbf{1 3}]$. In the work of Li-Liu-Liu-Zhou [20], following the earlier work on open string theory in $[\mathbf{2 1}]$, they envisioned that the topological vertex should be the degree of the virtual cycle of the moduli of relative stable morphisms to a toric Calabi-Yau containing three $\mathbb{P}^{1}$ intersecting at a single point, relative to the divisor the three other points on the three $\mathbb{P}^{1}$. Note that the topological types of such maps are given by their Euler characteristics and the winding numbers $\vec{\mu}$ at the relative divisor (the degree is determined by the winding numbers in this case.) The choice of the framing, interpreted in algebraic geometry, is the splitting type of the normal bundle to the three lines in the Calabi-Yau threefold.

More precisely, for any choice of $\mathbf{n}=\left(n_{1}, n_{2}, n_{3}\right)$, we form a pair $(\hat{Y}, \hat{D})$ of a formal relative toric Calabi-Yau threefold with an invariant divisor $\hat{D}$ such that (a) as set $\hat{Y}_{\text {set }}$ is the union of lines $C_{1}, C_{2}$ and $C_{3}$ intersecting at a single point; it can be the three axes of $\mathbb{C}^{3}$ compactified by adding three $\infty$ to the three lines; the divisor $\hat{D} \cap \hat{Y}_{\text {set }}$ is the three $\infty$ 's added; (b) the pair $(\hat{Y}, \hat{D})$ is Calabi-Yau in that $\wedge^{3} \Omega_{\hat{Y}}(\log \hat{D}) \cong \mathcal{O}_{\hat{Y}} ;$ (c) The normal bundle to $C_{i}$ in $\hat{Y}$ is $\mathscr{O}_{C_{i}}\left(n_{i}\right) \oplus \mathcal{O}_{C_{i}}\left(-n_{i}-1\right)$.

Note that $(\hat{Y}, \hat{D})$ being a relative Calabi-Yau forces the degree of the tangent bundle $T \hat{Y}$ along each $C_{i}$ to be 1 .

For a given choice of $\mathbf{n}$, we pick $(\hat{Y}, \hat{D})$ be as before. Then for any choice of $\chi$ and $\vec{\mu}$, we form the moduli space $\overline{\mathcal{M}}_{\chi}^{\bullet}(\hat{Y}, \vec{\mu})$ of relative stable morphisms to $\hat{Y}$, relative to $\hat{D}$, of not necessarily connected domains $X$ of $\chi\left(\mathcal{O}_{X}\right)=\chi / 2$, so that its contact order with $\hat{D}_{i}$ is given by $\mu_{i}$ in $\vec{\mu}=\left(\mu_{1}, \mu_{2}, \mu_{3}\right)$. Since $\hat{Y}$ is smooth, the moduli space is a formal Deligne-Mumford stack with a perfect obstruction theory $[\mathbf{1 8}]$.

To define the topological vertex of type $(\vec{\nu}, \mathbf{n})$, we further pick three $\left(\mathbb{C}^{*}\right)^{3}$-invariant divisors $\hat{L}_{i} \subset \hat{D}_{i}$ and form their union $\hat{L}=\cup_{i=1}^{3} \hat{L}_{i}$. We let

$$
\overline{\mathcal{M}}_{\chi}^{\bullet}(\hat{Y}, \vec{\mu}, \hat{L}) \subset \overline{\mathcal{M}}_{\chi}^{\bullet}(\hat{Y}, \vec{\mu})
$$

be the closed sub-stack that is the moduli of relative stable morphisms with additional constraint that the intersection of the image of the relative stable maps with $\hat{D}$ lie in $\hat{L}$. The two stacks share identical closed points; 
however their obstruction theories are different. The virtual dimension of $\overline{\mathcal{M}}_{\chi}^{\bullet}(\hat{Y}, \vec{\mu}, \hat{L})$ is zero.

We let $\left[\overline{\mathcal{M}}_{\chi}^{\bullet}(\hat{Y}, \vec{\mu}, \hat{L})\right]^{\text {vir }}$ be the virtual cycle of the perfect obstruction theory of $\overline{\mathcal{M}}_{\chi}^{\bullet}(\hat{Y}, \vec{\mu}, \hat{L})$. It is a dimension zero class. The mathematical definition of the topological vertex $C_{\vec{\mu}}(\lambda ; \mathbf{n})$ is the degree of $\left[\overline{\mathcal{M}}_{\chi}^{\bullet}(\hat{Y}, \vec{\mu}, \hat{L})\right]^{\text {vir }}$.

3.3. One difficult remains: since $\overline{\mathcal{M}}_{\chi}^{\bullet}(\hat{Y}, \vec{\mu}, \hat{L})$ is a formal DM-stack, the class $\left[\overline{\mathcal{M}}_{\chi}^{\bullet}(\hat{Y}, \vec{\mu}, \hat{L})\right]^{\text {vir }}$ is a formal class; its degree is not defined. To overcome this difficulty, we shall use the $\left(\mathbb{C}^{*}\right)^{3}$-localization to define its degree.

We pick a subgroup

$$
T \cong\left(\mathbb{C}^{*}\right)^{2} \subset\left(\mathbb{C}^{*}\right)^{3}
$$

that leaves the Calabi-Yau form $\omega \subset \Gamma\left(\wedge^{3} \Omega_{\hat{Y}}(\log \hat{D})\right)$ invariant. Since the pair $(\hat{Y}, \hat{D})$ is $T$-invariants, both the moduli space $\overline{\mathcal{M}}_{\chi}^{\bullet}(\hat{Y}, \vec{\mu}, \hat{L})$ and its obstruction theory are $T$-invariants, thus the cycle $\left[\overline{\mathcal{M}}_{\chi}^{\bullet}(\hat{Y}, \vec{\mu}, \hat{L})\right]$ is a $T$-equivariant cycle. We have

Definition-Theorem: We define

$$
C_{\vec{\mu}}(\lambda ; \mathbf{n})=\int_{\left[\overline{\mathcal{M}}_{\chi}^{\bullet}(\hat{Y}, \vec{\mu}, \hat{L})\right]^{\mathrm{vir}}} 1 \in \mathbb{Q}\left(u_{1} / u_{2}\right),
$$

where $u_{i}$ are the characters of the two copies of $\mathbb{C}^{*}$ in $T$. The integral $C_{\vec{\mu}}(\lambda ; \mathbf{n})$, which appriori it is a degree zero rational function in $u_{1}$ and $u_{2}$, is a constant. It only depend on $\chi, \vec{\mu}$ and $\mathbf{n}$. We call this rational number the relative $\mathrm{GW}$-invariants of the topological vertex $(\hat{Y}, \hat{D})$.

This constancy result is miraculous. It fails if we view $\left[\overline{\mathcal{M}}_{\chi}^{\bullet}(\hat{Y}, \vec{\mu}, \hat{L})\right]^{\text {vir }}$ as a $\left(\mathbb{C}^{*}\right)^{3}$-equivariant class. This constancy result strengthen the belief that for Calabi-Yau threefold, the open GW-invariants with boundaries on (special) Lagrangian submanifolds should be well-defined.

3.4. The constancy result holds true for general smooth relative toric Calabi-Yau threefold. Let $(W, V)$ be such a pair. To define its relative GWinvariants, one can mimic the case of $(\hat{Y}, \hat{D})$ by first forming the moduli of relative stable morphisms to $(W, V)$ and then take the degree of the $T$-equivariant virtual cycle of the moduli space. Here $T \subset\left(\mathbb{C}^{*}\right)^{3}$ is the two-dimensional torus fixing the Calabi-Yau form of $(W, V)$. The resulting integral of the $T$-equivariant virtual cycle is a rational number, independent of $u_{1} / u_{2}$.

To relate the relative $\mathrm{GW}$-invariants of $(W, V)$ to the topological vertex $\tilde{C}_{\vec{\mu}}(\lambda ; \mathbf{n})$, we need to degenerate the pair $(W, V)$ to a union of the elementary $(\hat{Y}, \hat{D})$ 's constructed before. The degeneration exists if we replace $W$ by the formal completion $\hat{W}$ of $W$ along the closure of the union of one-dimensional orbits of $W$, and replace $V$ by the divisor $\hat{V}=V \times_{W} \hat{W}$. 
The pairs $(\hat{W}, \hat{V})$ are examples of relative formal-toric-Calabi-Yau (FTCY) threefolds defined in [20]. In general, smooth relative FTCY threefold are constructed by a plenary trivalent graph with flags attached to the bivalent and univalent vertices. For a Toric Calabi-Yau, the graph is the image of one dimensional orbits under the moment map of $T$.

3.5. Introducing FTCY threefolds has two benefits:

Equivalence Theorem: Let $(W, V)$ be a smooth relative toric Calabi-Yau threefold and let $(\hat{W}, \hat{V})$ be its counterpart of relative FTCY threefold. The the relative $\mathrm{GW}$-invariants of $(W, V)$ coincides with the corresponding one of $(\hat{W}, \hat{V})$ defined using the localization formula.

The proof of this Theorem is a tautology. First, since $(W, V)$ is a toric relative Calabi-Yau threefold, its GW-invariants

$$
\operatorname{deg}\left[\overline{\mathcal{M}}_{\chi, d}(W, \vec{\mu})\right]^{\operatorname{vir}}
$$

(here $d$ is the degree of the maps consistent with the branching assignment $\vec{\mu})$ can be evaluated by the localization formula. On the other hand, a simple observation shows that the fixed loci

$$
\overline{\mathcal{M}}_{\chi, d}(W, \vec{\mu})^{T}=\overline{\mathcal{M}}_{\chi, d}(\hat{W}, \vec{\mu})^{T}
$$

and the obstruction near the fixed loci are identical. Thus the localization formula of the $T$-equivariant degrees of

$$
\operatorname{deg}_{T}\left[\overline{\mathcal{M}}_{\chi, d}(W, \vec{\mu})\right]^{T, \operatorname{vir}}=\operatorname{deg}_{T}\left[\overline{\mathcal{M}}_{\chi, d}(\hat{W}, \vec{\mu})\right]^{T, \operatorname{vir}}
$$

are identical.

Geometric Operation on FTCY: We can perform the following list of geometric operatios on FTCY threefolds: degeneration, smoothing, gluing and decomposition.

Let $(\hat{W}, \hat{V})$ be a FTCY threefold. Since $\hat{W}_{\text {set }}$ is a union of formal threefolds supported on $\mathbb{P}^{1}$ 's, we can pick one $\Sigma \cong \mathbb{P}^{1} \subset \hat{W}_{\text {set }}$ and degenerate $\hat{W}_{\text {set }}$ to $\hat{W}_{\text {set, } 1}$ union with $\hat{W}_{\text {set,2 }}$ by degenerating the chosen $\Sigma$ to a chain of two $\mathbb{P}^{1}$ 's. We denote the two $\mathbb{P}^{1}$ 's by $\Sigma_{1} \cup \Sigma_{2}$, agreeing that $\Sigma_{i} \subset W_{i}$.

To degenerate $\hat{W}$, we only need to give $\hat{W}_{\text {set,1 }}$ and $\hat{W}_{\text {set }, 2}$ formal scheme structures so that they are smooth formal toric Calabi-Yau threefolds. We let $l$ be the degree of the decomposition:

$$
N_{\Sigma / \hat{W}} \cong \mathscr{O}_{\Sigma}(l) \oplus \mathscr{O}_{\Sigma}(-l-2)
$$

For $\hat{W}_{1}$, away from $\Sigma_{1}$, it has the scheme structure of $\hat{W}$; along $\Sigma_{1}$, we choose an integer $a$ and make

$$
N_{\Sigma_{1} / \hat{W}_{1}} \cong \mathscr{O}_{\Sigma_{1}}(a) \oplus \mathscr{O}_{\Sigma_{1}}(-a-1)
$$

For $\hat{W}_{2}$, we do the same with $\hat{W}_{2}-\Sigma_{2}$ and choose

$$
N_{\Sigma_{2} / \hat{W}_{2}} \cong \mathscr{O}_{\Sigma_{2}}(l-a) \oplus \mathscr{O}_{\Sigma_{1}}(a-l-1) .
$$


It remains to glue $\hat{W}_{1}$ and $\hat{W}_{2}$ to make the union a degeneration of $\hat{W}$. We let $q=\Sigma_{1} \cap \Sigma_{2}$; we let $\hat{R}_{i}$ be the germ at the origin of the fiber $N_{\Sigma_{i} / \hat{W}_{i}} \mid q$. Note that with $\hat{V}_{i}$ be $\hat{V} \cap \hat{W}_{i} \cup \hat{R}_{i}$, then both

$$
\left(\hat{W}_{1}, \hat{V}_{1}\right) \text { and }\left(\hat{W}_{2}, \hat{V}_{2}\right)
$$

are relative formal toric Calabi-Yau threefolds. The gluing $\hat{W}_{1} \cup_{\hat{R}} \hat{W}_{2}$ is the result after gluing $\hat{W}_{1}$ and $\hat{W}_{2}$ along $\hat{R}_{1} \cong \hat{R}_{2}$ so that the factor $\mathscr{O}_{\Sigma}(l)$ degenerates to the gluing of $\mathscr{O}_{\Sigma_{1}}(a)$ and $\mathscr{O}_{\Sigma_{2}}(l-a)$. It is easy to see that the pair $(\hat{W}, \hat{V})$ degenerates to $\left(\hat{W}_{1} \cup_{\hat{R}} \hat{W}_{2}, \hat{V}\right)$.

The smoothing is the reverse of the degeneration. The process of gluing $\left(\hat{W}_{1}, \hat{V}_{1}\right)$ with $\left(\hat{W}_{2}, \hat{V}_{2}\right)$ along $\hat{R}_{1}=\hat{R}_{2}$ is the gluing; its converse is the decomposition.

3.6. Let the pairs $\left(\hat{W}_{i}, \hat{V}_{i}\right)$ be the result of degeneration followed by decomposition, then the degeneration formula of $\mathrm{GW}$-invariants of $[\mathbf{1 8}]$ implies that the generating function of the $\mathrm{GW}$-invariants of $(\hat{W}, \hat{V})$ takes the form

$$
F_{\chi, \vec{\mu}}^{\hat{W} \cdot}=\sum_{\chi_{1}+\chi_{2}-2 \ell(\nu)=\chi} F_{\chi 1, \vec{\mu}_{1} \cup \nu}^{\hat{W}_{1}} \cdot z_{\nu} \cdot F_{\chi_{2}, \vec{\mu}_{2} \cup \nu}^{\hat{W}_{2}} \cdot
$$

Here $\bullet$ stands for GW-invariants of not necessarily connected domains; the symbol $z_{\nu}$ is some combinatorial factor; $\vec{\mu}_{i}$ is the part of the partitions in $\vec{\mu}$ that associate to the relative divisor of $\hat{W}_{i}$ and $\nu$ is the partition associated to the relative divisor $\hat{R}_{1}=\hat{R}_{2}$ of length $\ell(\nu)$.

Since we can degenerate any $\mathbb{P}^{1}$ in a FTCY, by successively performing the same technique, we can degenerate any FTCY threefold $(\hat{W}, \hat{V})$ to a union of $k$ topological vertex $(\hat{Y}, \hat{D})$ constructed earlier. Repeatedly applying the degeneration formula, we can express the relative GW-invariant of $(\hat{W}, \hat{V})$ as summation over all topological vertex $C_{\chi, \vec{\mu}}(\lambda, \mathbf{n})$ that takes the form

$$
F_{\chi, \vec{\mu}}^{\hat{W} \cdot}=\sum C_{\vec{\mu}_{1}}\left(\lambda, \mathbf{n}_{k}\right) \cdot z \cdot \cdots z \cdot C_{\vec{\mu}_{k}}\left(\lambda, \mathbf{n}_{k}\right)
$$

The summation is over a group of combinatoric date determined by the degeneration data of $(\hat{W}, \hat{V})$. This is the gluing algorithms described in the topological vertex theory of $[\mathbf{1}]$.

This gives an explicit formula of all formal relative GW-invariants of relative formal toric Calabi-Yau threefolds, which include the GW-invariants of all toric Calabi-Yau threefolds, in terms of the simple ones $C_{\chi, \vec{\mu}}(\lambda, \mathbf{n})$.

3.7. Computationally, it is significantly simpler than the traditional algorithm of localization by summing over trees of the all curves types: given a toric Calabi-Yau threefold, the generating function of its GW-invariants in all genera in a fixed degree from summing over the trees gives rise to an infinite series. By this formula, this infinite series is equal to a finite sum in terms of symmetric functions. This simplification in part is from the invariance theorem, which implicitly implies many vanishing results. 
The summation further simplifies by the explicit form of the topological vertex $C_{\chi, \vec{\mu}}(\lambda, \mathbf{n})$ based on physically derived duality between $\mathrm{GW}$-invariants with Chern-Simons invariants of links. This explicit form is recently proved by Maulik-Oblomkov-Okounkv-Pandharipande [29]. This completes the mathematical theory of topological vertex theory on effectively computing all genera (relative) GW-invariants of smooth toric Calabi-Yau threefolds.

\section{On positive genera GW-invariants}

Another recent progress in Gromov-Witten theory is the proof by Li-Zinger $[\mathbf{2 2}]$ of the hyperplane theorem for (reduced) genus one GWinvariants of complete intersections and the proof by Zinger [40] of a conjectural formula for the genus one GW-invariants of quintic threefolds (degree 5 hypersurfaces in $\mathbf{P}^{4}$ ).

4.1. The celebrated formula of Candelas et al. [7] is a closed formula for genus zero GW-invariants of quintic threefolds. They derived this formula via mirror symmetry by studying the variation of Hodge structures on the mirror quintic. Bershadsky-Cecotti-Ooguri-Vafa [6] generalized this to the so-called Kodaira-Spencer theory of gravity. This theory provides a machinery that determines closed formulas for all genera GW-invariants of Calabi-Yau threefolds up to a finite number of indeterminancies.

For the quintic threefolds, they derived a conjectural formula for a genus one generating function. It takes the following form. Denote by $N_{g}^{d} \in \mathbb{Q}$ the genus $g$ degree $d \mathrm{GW}$-invariant of a quintic threefold. Let $I_{m}(q)$ be the degree $m$ polynomial defined by

$$
\sum_{m=0}^{\infty} I_{m}(q) w^{m} \equiv \sum_{d=0}^{\infty} q^{d} \frac{\prod_{r=1}^{r=5 d}(5 w+r)}{\prod_{r=1}^{r=d}(w+r)^{5}}
$$

and set

$$
Q=e^{I_{1}(q) / I_{0}(q)}, \quad J_{1}^{\prime}(q) \equiv\left\{1+\frac{d}{d q}\right\}\left(\frac{I_{1}(q)}{I_{0}(q)}\right) .
$$

The conjectured genus one formula $[\mathbf{6}$, eq. (24)] is then equivalent to

$$
2 \sum_{d=1}^{\infty} N_{1}^{d} Q^{d T}=\frac{25}{6} \ln Q+\ln \left(I_{0}(q)^{-62 / 3}\left(1-5^{5} q\right)^{-1 / 6} J_{1}^{\prime}(q)^{-1}\right) .
$$

In this notation, the genus zero formula for the quintic [7, eq. (5.13)] is equivalent to

$$
\sum_{d=1}^{\infty} N_{0}^{d} Q^{d}=-\frac{5}{2}\left(\left(\frac{I_{3}(q)}{I_{0}(q)}\right)-\left(\frac{I_{1}(q)}{I_{0}(q)}\right)\left(\frac{I_{2}(q)}{I_{0}(q)}\right)+\frac{1}{3}\left(\frac{I_{3}(q)}{I_{0}(q)}\right)^{3}\right) .
$$

The last formula was proved in the mid 1990's by Givental [10] and by Lian-Liu-Yau [26] independently. Both of their proofs commence from the hyperplane property for genus zero GW-invariants and use localization by a torus action. This hyperplane property transforms many genus 0 
GW-invariants of complete intersections into twisted GW-invariants of the ambient projective space; the localization is a computational tool that reduces the computition of the twisted GW-invariants of $\mathbb{P}^{n}$ to a very intricate combinatoric problem. This problem was solved by analyzing properties of a generating function for equivariant twisted genus zero GW-invariants of $\mathbf{P}^{n}$.

4.2. The hyperplane property was proposed by Kontsevich [16]; its proof follows from the work of $[\mathbf{5}, \mathbf{2 3}, \mathbf{2 4}]$. In order to simplify the discussion, we restrict the description here to projective hypersurfaces. Let $Y \subset \mathbb{P}^{n}$ be a smooth hypersurface of degree $r$. For any degree $d$, we form the moduli space of genus $g$ stable morphisms to $Y$ and to $\mathbb{P}^{n}$, of degree $d$; both are DM-stacks, and since $Y \subset \mathbb{P}^{n}$ the former is a closed substack of the latter:

$$
\overline{\mathcal{M}}_{g}(Y, d) \subset \overline{\mathcal{M}}_{g}\left(\mathbb{P}^{n}, d\right)
$$

This inclusion can easily be defined as the vanishing of a global section. Let

$$
f: \mathcal{X} \longrightarrow \mathbb{P}^{n}, \quad \pi: \mathcal{X} \longrightarrow \overline{\mathcal{M}}_{g}\left(\mathbb{P}^{n}, d\right)
$$

be the universal family of $\overline{\mathcal{M}}_{g}\left(\mathbb{P}^{n}, d\right)$. The section $s \in \Gamma\left(\mathscr{O}_{\mathbb{P}}(r)\right)$ defining the hypersurface $Y=(s=0)$ induces a section $\tilde{s}=\pi_{*} f^{*}(s)$ of the sheaf

$$
\pi_{*} f^{*} \mathscr{O}_{\mathbb{P}^{n}}(r) \longrightarrow \overline{\mathcal{M}}_{g}\left(\mathbb{P}^{n}, d\right)
$$

It is easy to see that

$$
\overline{\mathcal{M}}_{g}(Y, d)=(\tilde{s}=0) \subset \overline{\mathcal{M}}_{g}\left(\mathbb{P}^{n}, d\right) .
$$

This inclusion immediately leads to the hyperplane property for genus zero GW-invariants. Since $\mathbb{P}^{n}$ has ample tangent bundle, $\overline{\mathcal{M}}_{0}\left(\mathbb{P}^{n}, d\right)$ is a smooth DM-stack of dimension $(n+1) d+1$. The obstruction theory of $\overline{\mathcal{M}}_{0}(Y, d)$ induced by the defining equation $\tilde{s}=0$ coincides with the standard obstruction theory of $\overline{\mathcal{M}}_{0}(Y, d)$. Consequently, the push-forward of the virtual cycle $\left[\overline{\mathcal{M}}_{0}(Y, d)\right]^{\text {vir }}$ in $\overline{\mathcal{M}}_{0}\left(\mathbb{P}^{n}, d\right)$ is the Euler class $e\left(\pi_{*} f^{*} \mathscr{O}(r)\right)$. In the case of a quintic threefold $Y_{5}$, we obtain the numerical relation

$$
N_{0}^{d}=\int_{\left[\overline{\mathcal{M}}_{0}\left(Y_{5}, d\right)\right]^{\mathrm{vir}}} 1=\int_{\overline{\mathcal{M}}_{0}\left(\mathbb{P}^{4}, d\right)} e\left(\pi_{*} f^{*} \mathscr{O}(5)\right) .
$$

Note that the right hand side of (4.3) is the integration of a tautological class against the (virtual) cycle $\left[\overline{\mathcal{M}}_{0}\left(\mathbb{P}^{4}, d\right)\right]$; it is a twisted $\mathrm{GW}$-invariant of $\mathbb{P}^{4}$.

In generalizing the hyperplane property to the positive genus case, we face the difficulty that $\overline{\mathcal{M}}_{g}\left(\mathbb{P}^{n}, d\right)$ is singular for $g \geq 1$ and the sheaf $\pi_{*} f^{*} \mathscr{O}(r)$ is not locally free. Replacing the Euler class by the Chern class of the $K$-class $R^{\bullet} \pi_{*} f^{*} \mathscr{O}(r)$ is not a solution for $g \geq 1$. 
4.3. In 2004, Li-Zinger [22] proved that a hyperplane property holds for reduced genus one GW-invariants of complete intersections. It is evident from their definition in $[\mathbf{3 9}]$ that the reduced genus one $\mathrm{GW}$-invariants differ from the standard ones by some combination of genus $\mathrm{GW}$-invariants. An explicit formula for the difference is given in [41]. It takes a particularly simple form in dimensions 2 and 3 as already observed in [39]. In the case of a quintic threefold $Y_{5}$ (or any Calabi-Yau threefold),

$$
N_{1}^{d}=N_{1}^{d, r e d}+\frac{1}{12} N_{0}^{d}
$$

where $N_{1}^{d, r e d} \in \mathbb{Q}$ is the reduced genus one degree $d$ GW-invariant of $Y_{5}$.

TheOREM (Li-Zinger [22]). The reduced genus one $G W$-invariants of a complete intersection involving only restrictions of cohomology classes from the ambient projective space (and descendant invariants) equal to certain twisted reduced genus one $G W$-invariants of the ambient projective space.

In light of the hyperplane property for genus zero GW-invariants, this theorem is essentially equivalent to a hyperplane property for the ordinary genus one GW-invariants of complete intersections. It is applied by Zinger $[\mathbf{4 0}]$ to confirm the BCOV prediction for the genus one GW-invariants of a quintic threefold.

TheOREM (Zinger [40]). The genus one GW-invariants of a quintic threefold are given by (4.1).

4.4. The moduli space $\overline{\mathcal{M}}_{1}\left(\mathbb{P}^{n}, d\right)$ splits into two parts: one consists of stable morphisms $u: C \rightarrow \mathbb{P}^{n}$ whose restrictions to the smallest genus one subcurve $C_{e} \subset C$ are non-constant; the other consists of those such that $\left.u\right|_{C_{e}} \equiv$ const.. (In the later case, we say that $u$ has a ghost elliptic subcurve.) The first part is a Zariski open subset of $\overline{\mathcal{M}}_{1}\left(\mathbb{P}^{n}, d\right)$; it is smooth and irreducible of dimension $(n+1) d$. Its closure

$$
\overline{\mathcal{M}}_{1}\left(\mathbb{P}^{n}, d\right)_{0} \subset \overline{\mathcal{M}}_{1}\left(\mathbb{P}^{n}, d\right),
$$

called the primary component of $\overline{\mathcal{M}}_{1}\left(\mathbb{P}^{n}, d\right)$, is singular though.

Roughly speaking, the standard GW-invariants arise from the moduli spaces $\overline{\mathcal{M}}_{g}\left(\mathbb{P}^{n}, d\right)$, while the reduced genus one $\mathrm{GW}$-invariants arise just from the primary component $\overline{\mathcal{M}}_{1}\left(\mathbb{P}^{n}, d\right)_{0}$ of $\overline{\mathcal{M}}_{1}\left(\mathbb{P}^{n}, d\right)$. They are obtained by integration of natural cohomology classes against the virtual class of the "primary component"

$$
\overline{\mathcal{M}}_{1}(Y, d)_{0} \subset \overline{\mathcal{M}}_{1}(Y, d)
$$

of the moduli space of stable maps into $Y$. If $Y \subset \mathbb{P}^{n}$,

$$
\overline{\mathcal{M}}_{1}(Y, d)_{0}=\overline{\mathcal{M}}_{1}(Y, d) \cap \overline{\mathcal{M}}_{1}\left(\mathbb{P}^{n}, d\right)_{0} \subset \overline{\mathcal{M}}_{1}\left(\mathbb{P}^{n}, d\right) ;
$$

this definition of $\overline{\mathcal{M}}_{1}(Y, d)_{0}$ is independent of the embedding $Y \hookrightarrow \mathbb{P}^{n}$.

The sheaf (4.2) is the sheaf of sections of the morphism

$$
p: \overline{\mathcal{M}}_{g}(\mathcal{L}, d) \longrightarrow \overline{\mathcal{M}}_{g}\left(\mathbb{P}^{n}, d\right),
$$


where $\mathcal{L}$ is the total space of the line bundle $\mathscr{O}_{\mathbb{P} n}(r)$ and $p$ is simply the composition with the projection map $\mathcal{L} \longrightarrow \mathbb{P}^{n}$. This sheaf is not locally free and $p$ is not a vector bundle if $g \geq 1$. Nevertheless, Zinger [38] shows that the restriction of $p$ to the primary component $\overline{\mathcal{M}}_{1}\left(\mathbb{P}^{n}, d\right)_{0}$ has a well-defined euler class, even though this restriction is still not a vector (orbi-)bundle (the rank of the fibers of $p$ jumps over the elements of $\overline{\mathcal{M}}_{1}\left(\mathbb{P}^{n}, d\right)_{0}$ with a ghost elliptic subcurve). In particular, the euler class of every desingularization of this restriction of (4.2) is essentially the same. It is shown in [22] that this euler class provides a hyperplane property for genus one $\mathrm{GW}$-invariants of complete intersections.

Theorem (Li-Zinger [22]). For any degree $r$ hypersurface $Y$ in $\mathbb{P}^{n}$, the image of $\left[\overline{\mathcal{M}}_{1}(Y, d)_{0}\right]^{\text {vir }}$ in the homology of $\overline{\mathcal{M}}_{1}\left(\mathbb{P}^{n}, d\right)_{0}$ is the Euler class of the restriction of the sheaf (4.2) to $\overline{\mathcal{M}}_{1}\left(\mathbb{P}^{n}, d\right)_{0}$ :

$$
\iota_{*}\left[\overline{\mathcal{M}}_{1}(Y, d)_{0}\right]^{\mathrm{vir}}=e\left(\pi_{0 *} f^{*} \mathscr{O}(r)\right) \cap\left[\overline{\mathcal{M}}_{1}\left(\mathbb{P}^{n}, d\right)_{0}\right] .
$$

In the case of a quintic threefold $Y_{5} \subset \mathbb{P}^{4}$, the relation (4.5) reduces to

$$
N_{1}^{d, \text { red }}=\int_{\overline{\mathcal{M}}_{1}\left(\mathbb{P}^{4}, d\right)_{0}} c_{5 d}\left(\pi_{*} f^{*} \mathscr{O}(5)\right) .
$$

The Atiyah-Bott localization theorem [3] then reduces the computation of (4.6) to integration over neighborhoods of the fixed loci of $\overline{\mathcal{M}}_{1}\left(\mathbb{P}^{4}, d\right)_{0}$ under the $\left(\mathbb{C}^{*}\right)^{5}$-action. The results of this computation for $d=2,3,4$ $\left(\overline{\mathcal{M}}_{1}\left(\mathbb{P}^{4}, 1\right)_{0}=\emptyset\right)$ were announced in $[\mathbf{2 2}]$ and agreed with the BCOV prediction (4.1).

An explicit desingularization of the primary component $\overline{\mathcal{M}}_{1}\left(\mathbb{P}^{n}, d\right)_{0}$ of $\overline{\mathcal{M}}_{1}\left(\mathbb{P}^{n}, d\right)$,

$$
\tilde{\mathcal{M}}_{1}\left(\mathbb{P}^{n}, d\right)_{0} \longrightarrow \overline{\mathcal{M}}_{1}\left(\mathbb{P}^{n}, d\right)_{0}
$$

is constructed by Vakil-Zinger [36]. Let

$$
\tilde{f}_{0}: \mathcal{X}_{0} \rightarrow \mathbb{P}^{n} \quad \text { and } \quad \tilde{\pi}_{0}: \mathcal{X}_{0} \rightarrow \tilde{\mathcal{M}}_{1}\left(\mathbb{P}^{n}, d\right)_{0}
$$

denote the pull back of the universal family on $\overline{\mathcal{M}}_{1}\left(\mathbb{P}^{n}, d\right)$. It was shown in [36] that the direct image sheaf

$$
\tilde{\pi}_{0 *} f_{0}^{*} \mathscr{O}(r) \longrightarrow \tilde{\mathcal{M}}_{1}\left(\mathbb{P}^{n}, d\right)_{0}
$$

is locally free of rank $r d$. On the other hand, (4.6) immediately leads to

$$
N_{1}^{d, \text { red }}=\int_{\tilde{\mathcal{M}}_{1}\left(\mathbb{P}^{4}, d\right)_{0}} c_{5 d}\left(\tilde{\pi}_{0 *} \tilde{f}^{*} \mathscr{O}(5)\right) .
$$

This re-statement of (4.6) is more computationally convenient as the localization theorem immediately reduces the right-hand side of (4.7) to integration over the $\left(\mathbb{C}^{*}\right)^{5}$-fixed loci in $\tilde{\mathcal{M}}_{1}\left(\mathbb{P}^{4}, d\right)_{0}$, rather than their neighborhoods. 
4.5. The original approach of Li-Zinger [22] is analytic, built on the delicate analysis of Zinger [39]. Roughly speaking, the reduced genus one invariants of $Y$ are first defined intrisically in [39] by deforming the CauchyRiemann equation. In the case $Y$ is a complete intersection in $\mathbb{P}^{n}$, it is then shown in [22] that the CR-equations for $Y$ and $\mathbb{P}^{n}$ can be deformed in consistent fashion. Furthermore, it is shown that the obstruction theory for the primary component of the moduli space of solutions of the deformed CR-equation for $Y$ viewed as the vanishing locus of the section $\tilde{s}$ coincides with its standard obstruction theory. This then implies the theorem.

In contrast to the hyperplane property for genus zero GW-invariants, the hyperplane property in genus one is far less immediate even assuming the restriction of the sheaf $(4.2)$ to $\tilde{\mathcal{M}}_{1}\left(\mathbb{P}^{n}, d\right)_{0}$ has a well-defined Euler class. In fact, the argument in [22] includes another, more robust, way of assembling the local analytic results of $[\mathbf{3 9}]$ to construct the Euler class.

Currently, Hu-Li [12] and Chang-Li [8] are developing an algebraic approach to reduced GW-invariants of complete intersections, the hyperplane property for them, and the splitting property (4.4) for Calabi-Yau complete intersections. The genus one case is almost complete; higher genus cases are promising. In the remainder of this section, we briefly outline the main ideas behind the algebraic construction in genus one and discuss possible generalizations to higher genus.

4.6. The first step is to reprove Vakil-Zinger's desingularization via an algebraic approach. To desingularize the moduli space $\overline{\mathcal{M}}_{1}\left(\mathbb{P}^{n}, d\right)$, HuLi [12] first describe a modular blowup of the moduli space of weighted nodal curves and then take a fiber product with $\overline{\mathcal{M}}_{1}\left(\mathbb{P}^{n}, d\right)$ to obtain the desired modular desingularization.

Definition. A weight on a nodal curve $C$ is an element $w \in H^{2}(C, \mathbb{Z})$ such that $w(\Sigma) \geq 0$ for every irreducible component $\Sigma \subset C$. A weighted nodal curve is a pair $(w, C)$ of a weight $w$ on a curve $C$.

We can make sense of a flat family of weighted nodal curves by requiring that the total weight on any flat family of closed subcurves be constant. We call a weighted nodal curve stable if every smooth ghost (i.e., weight 0) rational subcurve contains at least three nodes of the curve.

For any genus $g>0$, the moduli space $\mathfrak{M}_{g}^{w}$ of stable weighted nodal curves is a smooth Artin stack of dimension $3 g-3$. The projection $\mathfrak{M}_{g}^{w} \rightarrow$ $\mathfrak{M}_{g}$, where $\mathfrak{M}_{g}$ is the Artin stack of nodal genus $g$ curves, induced by forgetting the weight assignment is étale.

4.7. Each element $(w, C)$ either has a ghost-core or a non-ghost core curve. If $(w, C) \in \mathfrak{M}_{1}^{w}$, since $C$ has genus 1 , it has a smallest elliptic subcurve, denoted by $C_{e}$ and called the core curve of $C$. We say $(w, C)$ has a ghost-core curve if the restriction of $w$ to $C_{e}$ is zero. Otherwise, we say $(w, C)$ has a non-ghost core curve. 
The stack $\mathfrak{M}_{1}^{w}$ contains an open substack $\stackrel{\circ}{\Theta}_{0}$ of weighted curves with non-ghost core curves. The complement $\mathfrak{M}_{1}^{w} \backslash \stackrel{\odot}{\Theta}_{0}$ admits a natural partition according to the number of rational trees attached to the ghost core curve: $\stackrel{\circ}{\Theta}_{k}$ is the subset of pairs $(w, C)$ such that $C$ can be obtained from the ghost core $C_{e} \subset C$ by attaching $k$ (connected) trees of rational curves to $C_{e}$ at $k$ distinct smooth points of $C_{e}$. Then $\mathfrak{M}_{1}^{w}=\coprod_{k>0} \stackrel{\circ}{\Theta}_{k}$. We let $\Theta_{k}$ be the closure of $\stackrel{\circ}{\Theta}_{k}$.

We can successively blow up $\mathfrak{M}_{1}^{w}$ along the loci $\Theta_{k}$. $\Theta_{1}$ is a smooth Cartier divisor; blowing up along $\Theta_{1}$ does nothing. We then proceed to blow up $\mathfrak{M}_{1}^{w}$ along the locus $\Theta_{2}$, which is a smooth codimension 2 closed substack of $\mathfrak{M}_{1}^{w}$; we denote the resulting smooth stack by $\mathfrak{M}_{1,[2]}^{w}$. Inductively, after obtaining $\mathfrak{M}_{1,[k-1]}^{w}$, we blow it up along the proper transform of the closed substack $\Theta_{k} \subset \mathfrak{M}_{1}^{w}$. Since the proper transform is a smooth closed substack of $\mathfrak{M}_{1,[k-1]}^{w}$ of codimension $k$, the new stack is smooth. We continue this process for all $k \geq 1$. Since each connected component of $\mathfrak{M}^{w}$ is of finite type, the blowup process on each component will terminate after a finite number of steps. Therefore, the limit stack is a well-defined smooth Artin stack; we denote it by $\tilde{\mathfrak{M}}_{1}^{w}$.

4.8. The desingularization of $\overline{\mathcal{M}}_{1}\left(\mathbb{P}^{n}, d\right)$ is constructed via the fiber product

$$
\tilde{\mathcal{M}}_{1}\left(\mathbb{P}^{n}, d\right)=\overline{\mathcal{M}}_{1}\left(\mathbb{P}^{n}, d\right) \times_{\mathfrak{M}_{1}^{w}} \tilde{\mathfrak{M}}_{1}^{w}
$$

Here the morphism

$$
\overline{\mathcal{M}}_{1}\left(\mathbb{P}^{n}, d\right) \longrightarrow \mathfrak{M}^{w}
$$

is defined via the domain curves of the universal family $f: \mathcal{X} \rightarrow \mathbb{P}^{n}$ of $\overline{\mathcal{M}}_{1}\left(\mathbb{P}^{n}, d\right)$ together with the degree of the first Chern class $c_{1}\left(f^{*} \mathscr{O}_{\mathbb{P}^{n}}(1)\right)$.

Note that since $c_{1}\left(f^{*} \mathscr{O}_{\mathbb{P}^{n}}(1)\right)$ has degree $d$, defining $\tilde{\mathcal{M}}_{1}\left(\mathbb{P}^{n}, d\right)$ requires blowing up $\mathfrak{M}^{w}$ along the proper transforms of $\Theta_{k}$ from $k=2$ to $k=d$. That is,

$$
\tilde{\mathcal{M}}_{1}\left(\mathbb{P}^{n}, d\right)=\overline{\mathcal{M}}_{1}\left(\mathbb{P}^{n}, d\right) \times_{\mathfrak{M}_{1}^{w}} \mathfrak{M}_{1,[d]}^{w} .
$$

This recovers Vakil-Zinger's virtual blowup of $\overline{\mathcal{M}}_{1}\left(\mathbb{P}^{n}, d\right)$.

TheOrem (Vakil-Zinger $[\mathbf{3 6}]$, Hu-Li $[\mathbf{1 2}]) . \tilde{\mathcal{M}}_{1}\left(\mathbb{P}^{n}, d\right)$ is a DM-stack with smooth irreducible components that intersect transversally.

The direct image sheaves are regularized at the same time. Let

$$
\tilde{\mathcal{M}}_{1}\left(\mathbb{P}^{n}, d\right)_{0} \subset \tilde{\mathcal{M}}_{1}\left(\mathbb{P}^{n}, d\right)
$$

be the component associated to the primary component $\overline{\mathcal{M}}_{1}\left(\mathbb{P}^{n}, d\right)_{0}$ of $\overline{\mathcal{M}}_{1}$ $\left(\mathbb{P}^{n}, d\right)$. For each $k>0$, let

$$
\tilde{\mathcal{M}}_{1}\left(\mathbb{P}^{n}, d\right)_{k} \subset \tilde{\mathcal{M}}_{1}\left(\mathbb{P}^{n}, d\right)
$$

be the pre-image of

$$
\overline{\mathcal{M}}_{1}\left(\mathbb{P}^{n}, d\right)_{k}=\overline{\mathcal{M}}_{1}\left(\mathbb{P}^{n}, d\right) \times_{\mathfrak{M}_{1}^{w}} \Theta_{k}
$$


under the blow-down map. Let

$$
f_{k}: \mathcal{X}_{k} \longrightarrow \mathbb{P}^{n} \quad \text { and } \quad \tilde{\pi}_{k}: \mathcal{X}_{k} \longrightarrow \tilde{\mathcal{M}}_{1}\left(\mathbb{P}^{n}, d\right)_{k}
$$

be the pull back universal family.

Proposition $([\mathbf{3 6}, \mathbf{1 2}])$. The sheaves $\tilde{\pi}_{k *} f_{k}^{*} \mathscr{O}(r)$ are locally free on $\tilde{\mathcal{M}}_{1}\left(\mathbb{P}^{n}, d\right)_{k}$.

4.9. As shown in $[\mathbf{8}]$, this proposition leads directly to the hyperplane property for the reduced genus one GW-invariants of complete intersections.

For any complete intersection $Y \subset \mathbb{P}^{n}$, we define

$$
\begin{aligned}
\overline{\mathcal{M}}_{1}(Y, d)_{0} & =\overline{\mathcal{M}}_{1}(Y, d) \times \overline{\mathcal{M}}_{1}\left(\mathbb{P}^{n}, d\right) \\
\tilde{\mathcal{M}}_{1}(Y, d)_{0} & \left.=\overline{\mathcal{M}}_{1}(Y, d) \times \mathbb{P}^{n}, d\right)_{0}, \\
\overline{\mathcal{M}}_{1}\left(\mathbb{P}^{n}, d\right) & \tilde{\mathcal{M}}_{1}\left(\mathbb{P}^{n}, d\right)_{0} .
\end{aligned}
$$

If $Y$ is a Calabi-Yau threefold, the reduced genus one $\mathrm{GW}$-invariants of $Y$ should be the degree of the virtual cycle of $\overline{\mathcal{M}}_{1}(Y, d)_{0}$; this degree should be the same as the degree of $\tilde{\mathcal{M}}_{1}(Y, d)_{0}$, since the blowup construction is modular.

To define the virtual cycle we need a perfect obstruction theory. For $\overline{\mathcal{M}}_{1}(Y, d)_{0}$ and $\tilde{\mathcal{M}}_{1}(Y, d)_{0}$, we shall use the standard relative obstruction theories of

$$
\overline{\mathcal{M}}_{1}(Y, d)_{0} / \overline{\mathcal{M}}_{1}\left(\mathbb{P}^{n}, d\right)_{0} \quad \text { and } \quad \tilde{\mathcal{M}}_{1}(Y, d)_{0} / \tilde{\mathcal{M}}_{1}\left(\mathbb{P}^{n}, d\right)_{0} .
$$

This time, since $\overline{\mathcal{M}}_{1}\left(\mathbb{P}^{n}, d\right)_{0}$ is singular, the relative obstruction theory in the first case is not perfect. It is perfect in the second case.

4.10. Let $Y \subset \mathbb{P}^{n}$ a smooth hypersurface cut out by $s \in \Gamma\left(\mathscr{O}_{\mathbb{P}} n(r)\right)$ and let $\tilde{s}$ be the induced section of the sheaf:

$$
\tilde{\pi}_{0 *} \tilde{f}_{0}^{*} \mathscr{O}(r) \longrightarrow \tilde{\mathcal{M}}_{1}\left(\mathbb{P}^{n}, d\right)_{0}
$$

THEOREM. The relative obstruction theory of

$$
\tilde{\mathcal{M}}_{1}(Y, d)_{0} / \tilde{\mathcal{M}}_{1}\left(\mathbb{P}^{n}, d\right)_{0}
$$

induced from that of $\overline{\mathcal{M}}_{1}(Y, d)_{0} / \overline{\mathcal{M}}_{1}\left(\mathbb{P}^{n}, d\right)_{0}$ is perfect; this obstruction theory is identical to the one induced by the defining equation

$$
\tilde{\mathcal{M}}_{1}(Y, d)_{0}=(\tilde{s}=0) \subset \tilde{\mathcal{M}}_{1}\left(\mathbb{P}^{n}, d\right)_{0} .
$$

This theorem implies the hyperplane property (4.5). Thus, in the case $Y \subset \mathbb{P}^{4}$ is a quintic threefold, it immediately specializes to (4.7).

4.11. To generalize the construction of the reduced GW-invariants to higher genus via this approach, one needs to find a modular desingularization of the primary component of $\overline{\mathcal{M}}_{g}\left(\mathbb{P}^{n}, d\right)$ so that the lift of the relative obstruction theory of

$$
\overline{\mathcal{M}}_{g}(Y, d) / \overline{\mathcal{M}}_{g}\left(\mathbb{P}^{n}, d\right)
$$


is perfect. Since the relative obstruction theories with respect to the Artin stack of genus $g$ weighted nodal curves

$$
\overline{\mathcal{M}}_{g}(Y, d) / \mathfrak{M}_{g}^{w} \text { and } \quad \overline{\mathcal{M}}_{g}\left(\mathbb{P}^{n}, d\right) / \mathfrak{M}_{g}^{w}
$$

are well-understood, a modular desingularization obtained by blowing up $\mathfrak{M}_{g}^{w}$ is most desirable.

For $g=1$, this is the approach taken in $[\mathbf{2 2 , 3 6 ]}$. For $g=2$, the algebraic approach adopted in [12] can be generalized to obtain a modular desingularization of $\overline{\mathcal{M}}_{2}\left(\mathbb{P}^{n}, d\right)$. It remains to be seen how much higher one can push this approach.

ConjeCture. There is a modular desingularzation of the primary component $\overline{\mathcal{M}}_{g}\left(\mathbb{P}^{n}, d\right)_{0}$ of $\overline{\mathcal{M}}_{g}\left(\mathbb{P}^{n}, d\right), \tilde{\mathcal{M}}_{g}\left(\mathbb{P}^{n}, d\right)_{0} \rightarrow \overline{\mathcal{M}}_{g}\left(\mathbb{P}^{n}, d\right)_{0}$, with the following properties:

(i) if $\tilde{f}_{0}: \mathcal{X}_{0} \rightarrow \mathbb{P}^{n}$ and $\tilde{\pi}_{0}: \mathcal{X}_{0} \rightarrow \tilde{\mathcal{M}}_{g}\left(\mathbb{P}^{n}, d\right)_{0}$ is the pull-back of the universal family,

$$
\tilde{\pi}_{0 *} \tilde{f}_{0}^{*} \mathscr{O}_{\mathbb{P}^{n}}(r) \longrightarrow \tilde{\mathcal{M}}_{g}\left(\mathbb{P}^{n}, d\right)_{0}
$$

is locally free sheaf.

(ii) if $Y \subset \mathbb{P}^{n}$ is a smooth hypersurface cut out by $s \in \Gamma\left(\mathscr{O}_{\mathbb{P} n}(r)\right)$,

$$
\tilde{\mathcal{M}}_{g}(Y, d)_{0}=\overline{\mathcal{M}}_{g}(Y, d) \times \overline{\mathcal{M}}_{g}\left(\mathbb{P}^{n}, d\right) \tilde{\mathcal{M}}_{g}\left(\mathbb{P}^{n}, d\right)_{0} \subset \tilde{\mathcal{M}}_{g}\left(\mathbb{P}^{n}, d\right)_{0}
$$

is defined by the vanishing of the induced section $\tilde{s}=\tilde{\pi}_{0 *} \tilde{f}_{0}^{*}(s)$ of (4.8) and the induced relative obstruction theory of

$$
\tilde{\mathcal{M}}_{g}(Y, d)_{0} / \tilde{\mathcal{M}}_{g}\left(\mathbb{P}^{n}, d\right)_{0}
$$

coincides with the obstuction theory of the defining equation $\tilde{s}=0$.

Such a desingularization of $\overline{\mathcal{M}}_{g}\left(\mathbb{P}^{n}, d\right)_{0}$ would immediately give rise to reduced genus $g \mathrm{GW}$-invariants satisfying the hyperplane property

$$
\iota_{*}\left[\tilde{\mathcal{M}}_{g}(Y, d)_{0}\right]^{\mathrm{vir}}=e\left(\pi_{0 *} f^{*} \mathscr{O}(r)\right) \cap\left[\tilde{\mathcal{M}}_{g}\left(\mathbb{P}^{n}, d\right)_{0}\right],
$$

where $\iota: \tilde{\mathcal{M}}_{g}(Y, d)_{0} \longrightarrow \tilde{\mathcal{M}}_{g}\left(\mathbb{P}^{n}, d\right)_{0}$ is the inclusion. In the case of a quintic therefold $Y_{5} \subset \mathbb{P}^{4}$, this would reduce to

$$
N_{g}^{d, r e d}=\operatorname{deg}\left[\tilde{\mathcal{M}}_{g}\left(Y_{5}, d\right)_{0}\right]^{\text {vir }}=\int_{\left[\tilde{\mathcal{M}}_{g}\left(\mathbb{P}^{4}, d\right)_{0}\right]^{\text {vir }}} c_{*}\left(p_{0 *} f_{0}^{*} \mathscr{O}(5)\right) .
$$

4.12. We now outline an algebraic approach to the splitting property (4.4).

The failure of the hyperplane property for the ordinary genus one GWinvariants stems from the fact that the tautological homomorphism between the relative obstruction sheaves of

$$
\overline{\mathcal{M}}_{1}(Y, d) / \mathfrak{M}_{1} \quad \text { and } \quad \overline{\mathcal{M}}_{g}\left(\mathbb{P}^{n}, d\right) / \mathfrak{M}_{1}
$$


is not surjective. They are connected by the exact sequence

$$
\left.\left.\mathcal{O} b \overline{\mathcal{M}}_{1(Y, d)} \longrightarrow \mathcal{O} b{\overline{\mathcal{M}_{1}\left(\mathbb{P}^{n}, d\right)}}\right|_{\overline{\mathcal{M}}_{1}(Y, d)} \longrightarrow R^{1} \pi_{*} f^{*} \mathscr{O}(r)\right|_{\overline{\mathcal{M}}_{1}(Y, d)} \longrightarrow 0
$$

if $Y \subset \mathbb{P}^{n}$ is a smooth hypersurface of degree $r$.

The first arrow above is surjective if $g=0$ since $R^{1} \pi_{*} f^{*} \mathscr{O}(r)=0$. For $g=1$, it is surjective over a Zariski open subset of $\overline{\mathcal{M}}_{1}\left(\mathbb{P}^{n}, d\right)_{0}$; its cokernel is an invertible sheaf elsewhere. The failure of the surjectivity of the first arrow is accountable for the failure of the triple of the obstruction theory of $\overline{\mathcal{M}}_{1}(Y, d)$, the obstruction theory of $\overline{\mathcal{M}}_{1}\left(\mathbb{P}^{n}, d\right)$, and the relative obstruction theory of $\overline{\mathcal{M}}_{1}(Y, d) / \overline{\mathcal{M}}_{1}\left(\mathbb{P}^{n}, d\right)$ to be a "perfect triangle".

Our solution is to take the modular desingularizations $\tilde{\mathcal{M}}_{1}(Y, d) \subset \tilde{\mathcal{M}}_{1}$ $\left(\mathbb{P}^{n}, d\right)$ and construct a proper DM-stack $W$ in between

$$
\tilde{\mathcal{M}}_{1}(Y, d) \subset W \subset \tilde{\mathcal{M}}_{1}\left(\mathbb{P}^{n}, d\right)
$$

so that $\tilde{\mathcal{M}}_{1}\left(\mathbb{P}^{n}, d\right)_{0} \cap W=\tilde{\mathcal{M}}_{1}(Y, d)_{0}$ and the triple of the obstruction theory of $\tilde{\mathcal{M}}_{1}(Y, d)$, the obstruction theory of $W$, and the relative obstruction theory of $\tilde{\mathcal{M}}_{1}(Y, d) / W$ is a "perfect triangle".

Once such a $W$ is constructed, then an application of techniques in rational equivalence of virtual cycles $[\mathbf{2 3}, \mathbf{1 5}]$ leads to a rational equivalence between the normal cone $\mathcal{C}_{X}$ of $X=\tilde{\mathcal{M}}_{1}(Y, d)$ and the normal cone to $X$ in the normal cone $\mathcal{C}_{W}$,

$$
\mathcal{C}_{X} \sim \mathcal{C}_{X / \mathcal{C}_{W}}
$$

The explicit geometry of $W$ shows that $\mathcal{C}_{W}$ has an irreducible component supported on $W_{0}=\tilde{\mathcal{M}}_{1}(Y, d)_{0}$, while all other irreducible components are supported on irreducible components of the closure $W_{+}$of $W-W_{0}$. We write this decomposition as $\mathcal{C}_{W}=\mathcal{C}_{W_{0}} \cup \mathcal{C}_{W_{+}}$. Let $X_{0}=X \cap W_{0}$ and $X_{+}=X \cap W_{+}$. Then,

$$
\mathcal{C}_{X / \mathcal{C}_{W}}=\mathcal{C}_{X_{0} / \mathcal{C}_{W_{0}}} \cup \mathcal{C}_{X_{+} / \mathcal{C}_{W_{+}}}
$$

Applying the Gysin map 0!, which is intersecting with the zero section, to this identity and the above mentioned rational equivalence, we obtain an identity of cycle classes

$$
\left[\tilde{\mathcal{M}}_{1}(Y, d)\right]^{\text {vir }}=0^{!}\left(\mathcal{C}_{X_{0} / \mathcal{C}_{W_{0}}}\right)+0^{!}\left(\mathcal{C}_{X_{+} / \mathcal{C}_{W_{+}}}\right)
$$

On the other hand, since $\tilde{\mathcal{M}}_{1}(Y, d)$ is a modular blowup of $\overline{\mathcal{M}}_{1}(Y, d)$, the virtual cycle $\left[\tilde{\mathcal{M}}_{1}(Y, d)\right]^{\text {vir }}$ is the same as $\left[\overline{\mathcal{M}}_{1}(Y, d)\right]^{\text {vir }}$. Of the terms on the right hand side of $(4.9)$, the first is $\left[\tilde{\mathcal{M}}_{1}(Y, d)_{0}\right]^{\text {vir }}$. If $Y$ is a Calabi-Yau threefold, the second term is a multiple of the cycle $\left[\overline{\mathcal{M}}_{0}(Y, d)\right]^{\text {vir }}$. Taking the degree, we recover the splitting formula (4.4) for the genus one GWinvariants of complete intersection Calabi-Yau threefolds. 
4.13. The splitting relation (4.4) should extend to all genera, i.e. the ordinary GW-invariants should split into reduced ones, each of which satisfies a hyperplane property. In the case $Y$ is a Calabi-Yau threefold, the reduced invariants should be related to the ordinary ones through a linear relation as described below.

ConjeCture (Li-Zinger [22]). There are reduced $G W$-invariants in all genera each of which satisfies a hyperplane property. For a Calabi-Yau threefold and any second homology class d, they should satisfy a linear splitting property with universal constants $\beta_{i}^{j}$ :

$$
N_{g}^{d}=N_{g}^{d, r e d}+\beta_{g-1}^{g} N_{g-1}^{d, r e d}+\cdots+\beta_{0}^{g} N_{0}^{0, r e d} .
$$

The ideal case is if one can modular desingularize $\overline{\mathcal{M}}_{g}\left(\mathbb{P}^{n}, d\right)$; then the reduced invariants should be integrals of Euler classes over the virtual cycles of the primary component of $\overline{\mathcal{M}}_{g}\left(\mathbb{P}^{n}, d\right)$. This will be the hyperplane property of the reduced GW-invariants.

\section{Toward Gopakumar-Vafa's invariants}

The conjecture of Gopakumar-Vafa [11] on the virtual number of embedded curves and the recent conjecture of Maulik-Nekrasov-OkunkovPandharipande [28] and of Pandharipande-Thomas [30] on the the relation of GW-invariants and the Donaldson-Thomas (DT-)invariants shed a new light to the study of GW-invariants of Calabi-Yau threefolds.

As was mentioned earlier in this note, based on investigating string duality between type IIA and M-Theory, Gopakumar-Vafa conjectured that

Conjecture (Gopakumar-Vafa [11]). There are integers $n_{h}(\beta)$ such that the generating function of the $G W$-invariants of $X$ is of the form

$$
F_{X}(q, \lambda)=\sum_{h \geq 0, k>0, \beta \in H_{2}(X, \mathbb{Z})} n_{h}(\beta) \frac{1}{k}\left(2 \sin \frac{k \lambda}{2}\right)^{2 h-2} q^{k \beta} .
$$

Indeed, they argued that the collection $n_{h}(\beta)$ can be defined geometrically using the space of "D-branes". As suggested by many, the mathematical counter-part of D-branes should be stable sheaves of pure dimension one. Following this suggestion, to each class $\beta$ one can substitute the space of "D-branes" by the moduli $\mathcal{M}(\beta, 1)$ of pure dimension one stable sheaves $\mathcal{E}$ on $X$ with second Chern classes $c_{2}(\mathcal{E})=-\beta$ and Euler number $\chi(\mathcal{E})=1$. Then according to Gopakumar-Vafa, the number $n_{0}(\beta)$, which is the expected number of rational curves in $X$ of class $\beta$, should be the signed Euler number of $\mathcal{M}(\beta, 1)$ when it is smooth.

In case the moduli space is singular, since it has perfect obstruction theory $[\mathbf{3 5}]$, it has a dimension zero virtual cycle. The Gopakumar-Vafa conjecture suggests that the degree of the virtual cycle $[\mathcal{M}(\beta, 1)]^{\text {vir }}$ should be the number $n_{0}(\beta)$. 
Conjecture (Katz [14]). The number $n_{0}(\beta)$ is the degree of $[\mathcal{M}(\beta, 1)]^{\mathrm{vir}}$.

Part of this conjecture states that the degree of $[\mathcal{M}(\beta, 1)]^{\text {vir }}$ is independent of the choice of ample line bundle $H$ in defining the moduli space $\mathcal{M}(\beta, 1)$. The invariance of such number will follow if a wall-crossing formula for such moduli space is developed.

A different geometric definition of the numbers $n_{h}(\beta)$ is via the DTinvariants of ideal sheaves of Maulik-Nekrasov-Okounkov-Pandharipande [28] and of pairs of Pandharipande-Thomas [30].

Following [28], we let $I_{n}(X, \beta)$ be the Hilbert scheme of ideal sheaves $\mathscr{I}_{Z}$ of 1 -dimensional subscheme $Z \subset X$ such that $[Z]=\beta$ and $\chi\left(\mathscr{O}_{Z}\right)=n$. Viewing $\mathscr{I}_{Z}$ as a rank one torsion free sheaf, it is stable automatically. Following Thomas $[\mathbf{3 5}], I_{n}(X, \beta)$ has perfect obstruction theory. We define $I_{n, \beta}=\operatorname{deg}\left[I_{n}(X, \beta)\right]^{\text {vir }}$, define their partition function be

$$
Z_{D T, \beta}(q)=\sum_{n} I_{n, \beta} q^{n}
$$

and their reduced partition function

$$
Z_{D T, \beta}^{\prime}(q)=Z_{D T, \beta}(q) / Z_{D T, 0}(q) .
$$

The reduced DT-invariants $Z_{D T, \beta}^{\prime}$ is conjectural equivalent to the invariants of stable pairs. Following Thomas-Pandharipande [30], we consider the moduli $P_{n}(X, \beta)$ of pairs $\mathscr{O}_{X} \rightarrow \mathscr{E}$, where $\mathscr{E}$ is a pure dimension one sheaf of $c_{2}(\mathscr{F})=-\beta$ and $\chi(\mathscr{F})=n$, and $\mathscr{O}_{X} \rightarrow \mathscr{F}$ is stable in that the cokernel is a zero-dimensional sheaf. The moduli space of such pairs with fixed pair $(\beta, n)$ is a projective variety, proved by Le Potier $[\mathbf{1 7}]$.

By viewing the pair $\left[\mathscr{O}_{X} \rightarrow \mathscr{E}\right]$ as an element in the derived category of the coherent sheaves of $X$, Pandharipande-Thomas $[\mathbf{3 0}]$ proved that the deformation of the pair in $D^{b}(X)$ will remain of the form $\mathscr{O}_{X} \rightarrow \mathscr{E}^{\prime}$. This proves that the deformation of $\mathscr{O}_{X} \rightarrow \mathscr{E}$ is perfect (indeed symmetric). Therefore, the moduli space $P_{n}(X, \beta)$ has virtual cycle in dimension zero. Their degrees $P_{b, \beta}=\operatorname{deg}\left[P_{n}(X, \beta)\right]^{\text {vir }}$ form a partition function

$$
Z_{P, \beta}(q)=\sum_{n} P_{n, \beta} q^{n} .
$$

In [30], Pandharipande-Thomas conjectured that

Conjecture. The partition functions $Z_{P, \beta}(q)$ and $Z_{D T, \beta}^{\prime}(q)$ coincide, and after the transformation $-q=e^{i u}$ is equal to the partition function $Z_{G W, \beta}(u)$ of the disconnected $G W$-invariants $X$. Further, these series are rational function invariant under the transformation $q \rightarrow q^{-1}$.

Here the disconnected GW-invariants of $X$ for nonzero curve classes $\beta$ are

$$
Z_{G W, \beta}(u)=\sum_{g} N_{g, \beta}^{\bullet} u^{2 g-2}
$$


for $N_{g, \beta}^{\bullet}=\operatorname{deg}\left[\overline{\mathcal{M}}_{g}^{\bullet}(X, \beta)\right]^{\text {vir }}$, where $\overline{\mathcal{M}}_{g}^{\bullet}(X, \beta)$ is the moduli space of stable maps with possibly disconnected domains and no contracted connected components.

We remark that the conjecture is proved for $\beta$ a primitive class of $X[\mathbf{3 0}]$, and for toric Calabi-yau threefold [29]. In general, the conjecture implies that the Gopakumar-Vafa numbers $n_{g}(\beta)$ can be defined using the series $Z_{P, \beta}$ and will be integral since the degree $P_{n, \beta}$ are integral.

This is a fast progressing subject. A lot of questions remain open in this subject. For more details, please consult [30].

\section{Acknowledge}

The author thanks A. Zinger for helping the part on the analytic approach to the genus one GW-invariants of Calabi-Yau threefolds.

\section{References}

[1] M. Aganagic, A. Klemm, M. Marino, C. Vafa, The topological vertex, Comm. Math. Phys. 254 (2005), no. 2, 425-478.

[2] M. Aganagic, M. Marino, C. Vafa, All loop topological string amplitudes from ChernSimons theory, Comm. Math. Phys. 247 (2004), no. 2, 467-512.

[3] M. Atiyah and R. Bott, The moment map and equivariant cohomology, Topology 23 (1984), 1-28.

[4] K. Behrend, Gromov-Witten invariants in algebraic geometry, Invent. Math. 127 (1997), no. 3, 601-617.

[5] K. Behrend, B. Fantechi, Intrinsic normal cone, Invent. Math. 128 (1997), no. 1, 45-88.

[6] M. Bershadsky, S. Cecotti, H. Ooguri and C. Vafa, Holomorphic Anomalies in Topological Field Theories, Nucl. Phys. B405 (1993) 279-304; Kodaira-Spencer Theory of Gravity and Exact Results for Quantum String Amplitudes, Comm. Math. Phys. Volume 165, Number 2 (1994), 311-427.

[7] P. Candelas, X. dela Ossa, P. Green, and L. Parkes, A pair of Calabi-Yau manifolds as an exactly soluble superconformal theory, Nucl. Phys. B359 (1991) 21-74.

[8] H-L. Chang and J. Li, in preparation.

[9] K. Fukaya and K. Ono, Arnold conjecture and Gromov-Witten invariant, Topology 38 (1999), no. 5, 933-1048.

[10] A. Givental, Equivariant Gromov-Witten invariants, Internat. Math. Res. Notices 1996, no. 13, 613-663.

[11] R. Gopakumar and C. Vafa, M-Theory and Topological Strings-II. Journal of Mathematical Physics 42 (2001) 27-98.

[12] Y. Hu and J. Li, Genus-One Stable Maps, Local Equations and Vakil-Zinger's desingularization, preprint.

[13] E. Ionel and T. Parker, The symplectic sum formula for Gromov-Witten invariants, Ann. of Math. (2) 159 (2004), no. 3, 935-1025.

[14] S. Katz, Genus zero Gopakumar-Vafa invariants of contractible curves. math.AG/ 0601193.

[15] B. Kim, A. Kresch and T. Pantev, Functoriality in intersection theory and a conjecture of Cox, Katz, and Lee, J. Pure Appl. Algebra 179 (2003), no. 1-2, 127-136.

[16] M. Kontsevich, Enumeration of rational curves via torus actions, Commun. Math. Phys. 151 (1993), 245-262. 
[17] J. Le Potier, Faisceaux semi-stables et syst'emes coherents, In Vector bundles in algebraic geometry (Durham, 1993), Vol 208 of London Math. Soc. Lecture Note Ser., 179239. Cambridge Univ. Press: Cambridge, 1995.

[18] J. Li, Stable morphisms to singular schemes and relative stable morphisms, J. Differential Geom. 57 (2001), no. 3, 509-578; A degeneration formula of $G W$-invariants, J. Differential Geom. 60 (2002), no. 2, 199-293.

[19] A-M. Li and Y. Ruan, Symplectic surgery and Gromov-Witten invariants of CalabiYau 3-folds, Invent. Math. 145 (2001), no. 1, 151-218.

[20] J. Li, C.-C. Liu, K. Liu, J. Zhou, A Mathematical Theory of the Topological Vertex, to appear in Geom and Topology, math.AG/0408426.

[21] J. Li and Y. Song, Open string instantons and relative stable morphisms, Adv. Theor. Math. Phys. 5 (2001), no. 1, 67-91.

[22] J. Li and A. Zinger, On the Genus-One Gromov-Witten Invariants of a Quintic Threefold, math.AG/0406105.

[23] J. Li and G. Tian, Virtual moduli cycles and Gromov-Witten invariants of algebraic varieties, J. Amer. Math. Soc. 11 (1998), no. 1, 119-174.

[24] J. Li and G. Tian, Virtual moduli cycles and Gromov-Witten invariants of general symplectic manifolds, Topics in symplectic 4-manifolds (Irvine, CA, 1996), 47-83, First Int. Press Lect. Ser., I, Int. Press, Cambridge, MA, 1998.

[25] J. Li and G. Tian, Comparison of algebraic and symplectic Gromov-Witten invariants, Asian J. Math. 3 (1999), no. 3, 689-728.

[26] B. Lian, K. Liu and S.T. Yau, Mirror Principle I, Asian J. Math. Vol. 1, No. 4 (1997) 729-763.

[27] D. Morrison and R. Plesser, Summing the instantons, quantum cohomology and mirror symmetry in toric varieties, Nuclear Phys. B 440 (1995), no. 1-2, 279-354.

[28] D. Maulik, N. Nekrasov, A. Okounkov and R. Pandharipande, Gromov-Witten theory and Donaldson-Thomas theory I, Compos. Math. 142 (2006), no. 5, 1263-1285; Gromov-Witten theory and Donaldson-Thomas theory II, Compos. Math. 142 (2006), no. $5,1286-1304$.

[29] D. Maulik, A. Oblomkov, A. Okounkv, R. Pandharipande, Gromov-Witten/ Donaldson-Thomas correspondence for toric 3-folds, arXiv:0809.3976.

[30] R. Pandharipande and R. Thomas, Stable pairs and BPS invariants, arXiv:0711.3899; Curve counting via stable pairs in the derived category, arXiv:0707.2348.

[31] Y-B. Ruan, Gromov-Witten invariants and quantum cohomology, Geometric analysis and applications to quantum field theory (Adelaide, 1998/1999), 137-156, Progr. Math., 205, Birkhuser Boston, Boston, MA, 2002.

[32] Y-B. Ruan and G. Tian, A mathematical theory of quantum cohomology, J. Differential Geom. 42 (1995), no. 2, 259-367.

[33] B. Siebert, Symplectic Gromov-Witten invariants, New trends in algebraic geometry (Warwick, 1996), 375-424, London Math. Soc. Lecture Note Ser., 264, Cambridge Univ. Press, Cambridge, 1999.

[34] B. Siebert, Algebraic and symplectic Gromov-Witten invariants coincide, Ann. Inst. Fourier (Grenoble) 49 (1999), no. 6, 1743-1795.

[35] R. Thomas, A holomorphic Casson invariant for Calabi-Yau 3-folds, and bundles on K3 fibrations, Jour. Diff. Geom. 54 (2000) 367-438.

[36] R. Vakil and A. Zinger, A desingularization of the main component of the moduli space of genus-one stable maps into $\mathbb{P}^{n}$, Geom. Topol. 12 (2008), no. 1, 1-95.

[37] E. Witten, Two-dimensional gravity and intersection theory on moduli space, Surveys in differential geometry (Cambridge, MA, 1990), 243-310, Lehigh Univ., Bethlehem, PA, 1991.

[38] A. Zinger, On the structure of certain natural cones over moduli spaces of genus-one holomorphic maps, Adv. Math. 214 (2007) 878-933.

[39] A. Zinger, Reduced genus-one Gromov-Witten invariants, math/0507103. 
[40] A. Zinger, The reduced genus-one Gromov-Witten invariants of Calabi-Yau hypersurfaces, math/0705.2397, to appear in J. Amer. Math. Soc.

[41] A. Zinger, Standard vs. reduced genus-one Gromov-Witten invariants, Geom.\& Top 12 (2008), no. 2, 1203-1241.

[42] A. Zinger, Enumerative vs. symplectic invariants and obstruction bundles, J. Symplectic Geom. 2 (2004), no. 4, 445-543.

Department of Mathematics, Stanford University, Stanford, CA 94305, USA

E-mail address: jli@math.stanford.edu 
